\title{
Demographic influences on the performance of three memory tests by Sri Lankan older adults
}

\author{
Senadheera $C^{1}$, Pathirana KD ${ }^{2}$, Jayasekara KMSAK ${ }^{1}$, Kodituwakku PW $^{3}$, Dharmappriya SKCL ${ }^{2}$, \\ de Silva MLK ${ }^{2}$ \\ ${ }^{\prime}$ Department of Psychiatry, ${ }^{2}$ Department of Medicine, University of Ruhuna, Galle, Sri Lanka. \\ ${ }^{3}$ University School of Medicine, University of New Mexico, USA.
}

Correspondence: Dr. Chandanie Senadheera

e-mail: chandaniesenadheera@yahoo.co.uk

(1) https://orcid.org/0000-0003-3772-1365

\begin{abstract}
Introduction: Loss of memory is the hallmark of dementia. In Sri Lanka, validated tests are not available for assessment of memory. The objective of the project was to study performance of Sri Lankan older adults on three memory tests: Lankan Verbal Learning Test (LVLT), Benton Visual Retention Test (BVRT) and Modified Enhanced Cued Recall Test (MECRT).

Methods: Participants were 241 healthy individuals aged between 50 to 80 years. They were individually administered the tests by a research assistant. The LVLT is a verbal list learning task. The BVRT contains geometric figures that are presented in the recognition memory format. The MECRT assesses the memory for pictures aided with semantic cues.

Means of test scores were calculated and compared for gender, age and levels of education. Multiple linear regression models were used.

Results: The mean age was 63 ( $\mathrm{SD}=7.7)$ years. Gender effect was prominent on verbal memory scores. The test scores were influenced by gender and education level. Gender, age and education together explained $15.6 \%$ and $9.6 \%$ of the variance of verbal memory score and visual recognition memory score respectively. Variance of MECRT scores were minimally explained by demographic variables.

Conclusion: MECRT is the least influenced by demographic factors and could be used for Sri Lankan older persons irrespective of their gender, age and education.
\end{abstract}

Keywords: Dementia, demographic variables, memory tests, neuropsychological assessment, Sri Lanka

\section{Introduction}

Neuropsychological testing is an integral part in the assessment of neurocognitive disorders such as dementia, especially in the early stages. Dementia is characterised by amnesia with additional deficits in other cognitive domains. Therefore, assessing memory is important for making the diagnosis, development of intervention plans, monitoring disease progression and assessing effects of treatment. Many screening instruments and tests of different forms of memory (1) are being in wide use in developed countries but are scarce in developing countries such as Sri Lanka.

Sri Lanka has been rated as the fastest ageing population in South Asia (2). Country's share of population aged over 60 years will reach almost $30 \%$ by 2050 . In this backdrop prevalence of dementia is expected to rise in the country. 
Some tools for assessment of cognitive functions are validated for Sri Lankan population (3-9). Among them, the most frequently used tool is Sinhala version of Mini-Mental State Examination (MMSE). MMSE is a widely used screening tool throughout the world, it has most of the qualities of a good screening instrument described by Parker and Philip (10), but it assesses memory impairment only to a limited extent; it is also criticized for lack of specificity, particularly among less educated individuals. Therefore it is essential to develop and validate culture appropriate tests for assessing different forms, processes and operations of memory easily applicable for diverse sectors of Sri Lankan population including very old, illiterate and impaired individuals.

List learning tests such as Hopkins verbal learning test-revised (1) is a widely used measure of verbal learning and memory. It has been useful in detecting dementia; moreover according to Hogervorst (11), some of its scores found to be more sensitive than MMSE to the presence of dementia.

Enhanced cued recall test is a memory test which can be used with individuals with cognitive impairment including memory deficits as it uses pictures and provides cues to enhance storage and retrieval of information (12). This test has reported high sensitivity and specificity to discriminate demented from non-demented elderly (13).

Benton Visual Retention test is used to assess visual memory (14). It has different types of administration, including multiple choice version which is a measure of subject's visual recognition memory; it minimally relies on language.

Objective of our study was to compare performance on Lankan Verbal Learning Test, Modified Enhanced Cued Recall Test and Benton Visual Retention Test in a sample of healthy Sri Lankan older adults.

\section{Methods}

Our sample consisted of 241 randomly selected healthy subjects aged 50 to 80 years living in a semi urban community. Sample size was decided based on the sample sizes of previous such studies (8). Subjects were selected from randomly selected areas from cluster sampling method in a door to door survey in Bope-Poddala MOH area in Galle district which represents typical Sinhala speaking semi-urban community in southern part of the country. Medical records were reviewed and people with major psychiatric disorders, neurological conditions, uncontrolled diabetes, severe hearing or visual impairments were excluded. Potential subjects were screened for cognitive decline with Geriatric depression scale validated for Sri Lanka (6) and Modified version of the Short Form of Informant Questionnaire on Cognitive Decline and a brief functional screening instrument adapted to Sri Lanka (5). Those who did not meet criteria for depression and cognitive decline were invited to take part in the study. Neuropsychological tests were individually administered for each participant by a trained research assistant. Ethical approval for the study was obtained from the ethical review committee of Faculty of Medicine, University of Ruhuna.

\section{Lankan verbal learning test (LVLT)}

The LVLT is a verbal list learning task modeled after Hopkins verbal learning test-revised (15), in which participants were required to learn 12 words drawn from 3 categories over 3 learning trials. After each presentation of the list, the subject was asked to recall as many words s/he could. Following 20 minutes filled delay, the subject was asked for a free recall and in addition to recognise the words from a list with target words and distractors. Three measures of verbal memory are obtained from the test. The total recall score is the number of words recalled over the 3 trials (free recalls). To calculate the percent retained after the delay ( $\%$ retention), 20 minute delay recall is divided by the best of trial 2 and 3. The recognition discrimination index (RDI) is the number of true positives minus the number of false positives in the recognition trial.

\section{Modified Enhanced Cued Recall Test (MECRT)}

MECRT was originally developed by Grober, et al. (12) and in the current study, items of the original version were replaced with culturally appropriate items (eg: grapes replaced with bananas) but in the administration, procedures described in the original version were used. 
In MECRT line drawings of 16 items in four different cards (4 items in each) were presented to the participant. In the presentation of the first card, while the subject was examining the items on the card, examiner gave semantic cue for each item and asked the subject to name the matching drawing. If the subject correctly names it (Any variations of the dialect, including the English word were considered as correct. The participant is expected to produce the same name to indicate the particular item in his recalls), the examiner moves to the next item. After 4 items on the card were correctly named, the card is removed. Immediate recall was tested and the subject is asked to name each of the items after the same semantic cue is given by the examiner. This procedure was followed for all 4 cards. After all 4 cards were presented, the subject was asked to name as many items he/ she could recall (free recall); after the free recall, examiner provided semantic cues to help recall the remaining items (cued recall). After 20 minute filled gap, subject was again asked for a free recall of the items on the cards and cued recall of remaining items. In the analysis, number of items recalled over $1^{\text {st }}$ free recall, $1^{\text {st }}$ cued recall, delayed free recall, delayed cued recall, total number of free recalled items (in each of the 2 trials), total number of cued recall items (in each of the 2 trials) and total recalls were used. Total recall was the sum of free and cued recall items for each of the trials.

\section{Benton Visual Retention Test (BVRT)}

The administration $\mathrm{M}$ of the test was used. BVRT contains 15 geometric figures of varying complexity that are presented in the recognition memory format. Each item is shown for 10 seconds followed by a multiple choice page containing the target item and 3 foils. The participant is asked to select the target item (the one shown for 10 seconds) from among distracters. The number of correct responses was used as the measure of visual memory.

Analyses were conducted using Statistical Package of Social Science (SPSS) version 20. There is evidence that the genders differ in the performance on verbal memory tests. The sample was divided into two age categories; $50-64$ years and 65 years and above. Independent sample $\mathrm{T}$ test was used to compare mean test scores. The education levels were categorized as primary education (upto 5 years), 5-10 years of schooling and above 10 years of formal education. One-way ANOVA was used to compare test scores among age groups. Test scores were displayed as means, standard deviation for each gender, age category and education level. Mean scores within each group were compared and level of significance was set at .01 to control for inflated error rates.

Linear regression models were used to assess the effect of gender, age and education on tests scores simultaneously.

\section{Results}

The sample was dominated by females $(64 \%$, $\mathrm{n}=154)$. The mean age was $63(\mathrm{SD}=7.7)$ years. Twelve percent of the sample was either illiterate or had very little education. Nearly half of the sample reported their monthly income below poverty line. Demographic features of the sample are summarized in table 1.

All the participants $(\mathrm{N}=241)$ were able to perform all the 3 tests. A large majority of participants (92\%) achieved ceiling score for the two scores of MECRT; total number of recalls in each of the immediate recall and 20 minute delayed recall.

Analysis revealed that the test scores were influenced by gender and the years of formal education, but the influence of age on test scores was minimal. Gender has no effect on BVRT score but was prominent on all LVLT scores; females out performed males. Means of the test scores and their standard deviations for both males and females are summarized in Table 2 .

The mean test scores and standard deviations were calculated for test scores for two age groups. These data are provided in Table 3. Elderly group (aged above 64 years) demonstrated comparative levels of performance to their younger counterparts. As table 3 shows none of the differences of mean test scores in the two age groups were statistically different.

For analysis participants were divided into 3 groups based on their level of formal education. Mean test scores, and standard deviations were calculated for each of these 3 groups and these data are provided in Table 4. People with most years of 
education obtained better scores compared to those with least years of education.

Multiple linear regression model was used to learn effect of gender, age and education on test scores. In linear regression model, reference group for gender was the male group, for age it was the young group (50-64 years). The sample was divided in to two levels of education; up to 10 years and above 10 years of formal education. For education, the reference group was the group up to 10 years of education. The higher educated group was compared to the reference group.

Results of Multiple linear regression is shown in Table 5.

Gender, age and education together explain upto $16 \%$ of the varience in LVLT scores and almost $10 \%$ of the varience in BVRT score while varience in MECRT scores were least explained by gender, age and education level of the participants.
Table 1: Demographic characteristics of the sample

\begin{tabular}{lr}
\hline Variables & N (\%) \\
\hline Age (in years) & $140(58)$ \\
50 - 64 & $101(42)$ \\
$65-79$ & $154(64)$ \\
\hline Sex & $87(36)$ \\
$\quad$ Female & \\
Male & $28(12)$ \\
\hline Educational status & $142(59)$ \\
Upto 5 years & $71(29)$ \\
6 to 10 years & \\
More than 10 years & $60(25)$ \\
\hline Employment status & $115(48)$ \\
Employed & $66(27)$ \\
Retired/ Not working & \\
Never worked & $111(46)$ \\
\hline House-hold monthly income & $82(34)$ \\
Up to Rs. 10,000/- & $47(20)$ \\
Rs. 10,000/- to Rs. 20,000/- \\
More than Rs. 20,000/-
\end{tabular}

Table 2: Test scores for males and females; mean and standard deviation (SD) for 3 memory tests $(\mathrm{N}=241)$

\begin{tabular}{lccl}
\hline Test Score & $\begin{array}{l}\text { Males (n=87) } \\
\text { Mean (SD) }\end{array}$ & $\begin{array}{l}\text { Females (n=154) } \\
\text { Mean(SD) }\end{array}$ & P value \\
\hline LVLT & & & \\
Total recall & $18.8(3.2)$ & $21.7(3.7)$ & 0.001 \\
${ }^{*} \%$ retention & $72.4(22.8)$ & $80.6(18.1)$ & 0.002 \\
${ }^{* *}$ RDI & $7.1(.17)$ & $8.5(.14)$ & 0.001 \\
BVRT & $10.6(.24)$ & $10.2(.23)$ & 0.37 \\
\hline MCERT & & & \\
$1^{\text {st }}$ free recall & $8.2(1.9)$ & $8.6(2.1)$ & 0.151 \\
${ }^{* * *} 1^{\text {st }}$ cued recall & $7.7(1.9)$ & $7.3(2.1)$ & 0.180 \\
Delayed free recall & $8.5(2.2)$ & $9.3(2.4)$ & 0.010 \\
${ }^{* * *}$ delayed cued recall & $7.5(2.2)$ & $6.7(2.4)$ & 0.013 \\
Total free recall & $16.7(3.8)$ & $17.9(3.9)$ & 0.030 \\
Total cued recall & $15.1(3.7)$ & $14(3.9)$ & 0.024 \\
\hline
\end{tabular}

* one outlier removed for analysis

** as the distribution of was skewed, score was converted to square root for calculation of means

$* * *$ cues were given only for the items individual failed to freely recall. 
Table 3: Test scores for age: mean and standard deviation (SD) of scores of 3 memory tests $(\mathrm{N}=241)$

\begin{tabular}{|c|c|c|c|}
\hline \multirow[t]{3}{*}{ Test Score } & \multicolumn{2}{|c|}{ Age } & \multirow[t]{3}{*}{$P$ value } \\
\hline & Upto 64 yrs $(n=140)$ & Above 64 yrs $(n=101)$ & \\
\hline & Mean (SD) & Mean (SD) & \\
\hline \multicolumn{4}{|l|}{ LVLT } \\
\hline Total recall & $21.1 \quad(3.6)$ & $20.1 \quad(3.9)$ & 0.041 \\
\hline$* \%$ retention & $77.1 \quad(21.7)$ & 78.5 (18.3) & 0.604 \\
\hline$* * \mathrm{RDI}$ & $8.1 \quad(0.15)$ & $7.8(0.17)$ & 0.343 \\
\hline BVRT & $10.6 \quad(0.22)$ & $10.2 \quad(0.24)$ & 0.413 \\
\hline \multicolumn{4}{|l|}{ MECRT } \\
\hline $1^{\text {st }}$ free recall & $8.6 \quad(2.1)$ & $8.3 \quad(2.1)$ & 0.358 \\
\hline$* * * 1^{\text {st }}$ cued recall & $7.4 \quad(2.0)$ & $7.6(2.1)$ & 0.338 \\
\hline $1^{\text {st }}$ total recall & $15.9 \quad(0.31)$ & $15.9 \quad(0.3)$ & 0.537 \\
\hline Delayed free recall & $9.3(2.3)$ & $8.6 \quad(2.6)$ & 0.022 \\
\hline ***Delayed cued recall & $6.7 \quad(2.2)$ & $7.3(2.5)$ & 0.051 \\
\hline Total free recall & $17.9 \quad(3.8)$ & $16.9 \quad(4.1)$ & 0.059 \\
\hline Total cued recall & $14.1 \quad(3.6)$ & $14.9 \quad(4.1)$ & 0.084 \\
\hline
\end{tabular}

*one outlier removed for the analysis

**scores were converted in to square root for calculations as the distribution was skewed

$* * *$ cues were given only for the items individual failed to freely recall

Table 4: Test scores for level of education: mean and standard deviation (SD) of scores 3 memory tests $(\mathrm{N}=241)$

\begin{tabular}{|c|c|c|c|c|}
\hline \multirow[t]{2}{*}{ Test Score } & \multicolumn{3}{|c|}{ Years of education } & \multirow[t]{2}{*}{$P$ value } \\
\hline & $\begin{array}{c}\text { Upto } 5 \text { yrs. }(n=28) \\
\text { (Sean }\end{array}$ & 6 to 10 yrs. $(n=142)$ & $\begin{array}{c}\text { Above } 10 \text { yrs. }(n=71) \\
\text { Mean }(\mathrm{SD})\end{array}$ & \\
\hline \multicolumn{5}{|l|}{ LVLT } \\
\hline Total recall & $18.9 \quad(2.9)$ & $20.8 \quad(3.8)$ & $21.1 \quad(3.9)$ & 0.023 \\
\hline$* \%$ retention & $78 \quad(22.5)$ & $76.2(20.2)$ & $80.6(19.6)$ & 0.318 \\
\hline$* * \mathrm{RDI}$ & $6.5(0.25)$ & $8.1 \quad(0.14)$ & $8.4(0.12)$ & 0.001 \\
\hline BVRT & $(0.41)$ & $10.4 \quad(0.17)$ & $12.3(0.12)$ & 0.001 \\
\hline \multicolumn{5}{|l|}{ MECRT } \\
\hline $1^{\text {st }}$ free recall & $7.2(2.3)$ & $8.6 \quad(2.0)$ & $8.6 \quad(1.9)$ & 0.002 \\
\hline$* * * 1^{\text {st }}$ cued recall & $8.6(2.1)$ & $7.3(2.0)$ & $7.3(1.9)$ & 0.004 \\
\hline $1^{\text {st }}$ total recall & $15.8 \quad(0.5)$ & $15.9 \quad(0.3)$ & $15.9 \quad(0.3)$ & 0.182 \\
\hline Delayed free recall & $8.1 \quad(2.7)$ & $9 \quad(2.4)$ & $9.2(2.2)$ & 0.139 \\
\hline$* * *$ Delayed cued recall & $7.7 \quad(2.5)$ & $6.9 \quad(2.3)$ & $6.8 \quad(2.3)$ & 0.172 \\
\hline Delayed total recall & $15.9(0.4)$ & $15.9 \quad(0.3)$ & $15.9 \quad(0.3)$ & 0.397 \\
\hline Total free recall & $15.3 \quad(4.4)$ & $17.7 \quad(3.9)$ & $17.8 \quad(3.6)$ & 0.009 \\
\hline Total cued recall & 16.4 (4) & $14.2(3.8)$ & $14.1 \quad(3.7)$ & 0.017 \\
\hline
\end{tabular}

*one outlier removed for the analysis

**scores were converted in to square root for calculations as the distribution was skewed

$* * *$ cues were given only for the items individual failed to freely recall 
Table 5: Results of Multiple linear regression; association of scores of 3 memory tests with gender, age and education $(\mathrm{N}=241)$

\begin{tabular}{|c|c|c|c|c|c|c|c|c|}
\hline Score & Covariate & Coefficient & SE & $T$ & $P$ value & $95 \% \mathrm{CI}$ & $r$ coef. & Adj. $R^{2}$ \\
\hline$\overline{\text { LVLT }}$ & & & & & & & & \\
\hline Total recall & Gender & 2.945 & .465 & 6.328 & 0.001 & 2.028 & 3.862 & $15.7 \%$ \\
\hline & Age & -1.024 & .454 & -2.256 & 0.025 & -1.98 & -.130 & \\
\hline & Education & .834 & .491 & 1.697 & 0.091 & -.134 & 1.801 & \\
\hline$\%$ retention & Gender & 8.256 & 2.684 & 3.077 & 0.002 & 2.970 & 13.543 & $3.6 \%$ \\
\hline & Age & 1.248 & 2.612 & .478 & 0.633 & -3.898 & 6.394 & \\
\hline & Education & 4.072 & 2.837 & 1.435 & 0.152 & -1.517 & 9.661 & \\
\hline RDI & Gender & .259 & .051 & 5.091 & 0.001 & .159 & .360 & $10.6 \%$ \\
\hline & Age & -.048 & .050 & -.976 & 0.330 & -.146 & .049 & \\
\hline & Education & .119 & .054 & 2.198 & 0.029 & .012 & .225 & \\
\hline BVRT & Gender & -.057 & .061 & -.929 & 0.354 & -.178 & .064 & $9.6 \%$ \\
\hline & Age & -.079 & .060 & -1.313 & 0.190 & -.196 & .039 & \\
\hline & Education & .333 & .065 & 5.145 & 0.001 & .205 & .460 & \\
\hline MECRT & & & & & & & & \\
\hline $1^{\text {st }}$ free recall & Gender & .396 & .276 & 1.437 & 0.152 & -.147 & .939 & $0.3 \%$ \\
\hline & Age & -.257 & .269 & -.958 & .339 & -.787 & .272 & \\
\hline & Education & .245 & .291 & .843 & .400 & -.328 & .818 & \\
\hline $1^{\text {st }}$ cued recall & Gender & -.364 & .272 & -1.340 & 0.182 & -.898 & .171 & $0.1 \%$ \\
\hline & Age & .263 & .265 & .994 & 0.321 & -.259 & .785 & \\
\hline & Education & -.228 & .287 & -.794 & 0.428 & -.792 & .337 & \\
\hline Delayed free & Gender & .744 & .320 & 2.322 & 0.021 & .113 & 1.375 & $3.4 \%$ \\
\hline recall & Age & -.734 & .312 & -2.351 & 0.020 & -1.350 & .119 & \\
\hline & Education & .279 & .338 & .824 & 0.0411 & -.387 & .945 & \\
\hline Delayed cued & Gender & -.776 & .309 &.-2.510 & 0.013 & -1.384 & -.167 & $3.3 \%$ \\
\hline recall & Age & .607 & .301 & 2.015 & 0.045 & .014 & 1.201 & \\
\hline & Education & -.314 & .326 & -.962 & 0.337 & -.956 & .329 & \\
\hline Total free & Gender & .1 .140 & .522 & 2.184 & 0.030 & .111 & 2.168 & $2.6 \%$ \\
\hline recall & Age & -.992 & .509 & -1.948 & 0.053 & -1.995 & .011 & \\
\hline & Education & .524 & .551 & .951 & 0.343 & -.562 & 1.609 & \\
\hline Total cued & Gender & -1.162 & .511 & -2.275 & 0.024 & -2.169 & -.156 & $2.5 \%$ \\
\hline recall & Age & .889 & .498 & 1.784 & 0.076 & -.093 & 1.870 & \\
\hline & Education & -.515 & .539 & -.954 & 0.341 & -1.577 & .548 & \\
\hline
\end{tabular}

\section{Discussion}

Our study demonstrates that the three memory tests could be applied even to those without any formal education.

We observed that all test scores of LVLT are higher in females. This finding is consistent with the findings of other studies which report female superiority of verbal/ language based test performance (16). Our results revealed that the percent retention score is influenced only by gender but not by age or education. In contrast verbal recognition memory (RDI score) found to be influenced by gender as well as level of education. Female gender and having more years of education lead to better accuracy of the verbal recognition memory.
Memory decline with ageing is considered to be normal $(17,18)$. We observed slightly better performance in some scores by the group who is less aged. However our study reveals that performance of these three memory tests are not influenced by age in cognitively normal older adults.

Effects of education is marked on the test scores; those with little formal education performed poorly. This effect was prominent on free recall scores, but it does not have the effect on aided memory scores; that is those with little or no formal education performed as well as those with several years of formal education when encoding and retrieval of information are facilitated with semantic cues. 
Multiple linear regression analysis showed that a large proportion of variance $(10 \%)$ in visual memory score was explained by gender, age and education. This finding contradicts the well-known fact that non-verbal tests are culture free. Our results indicate that those with no or primary education scored low for BVRT. Therefore we believe that clinicians should be cautious in using this test for illiterate/ poorly educated subjects and in making interpretations of their score. In contrast, ECRT demonstrated to be a culture free test which could be used with older Sri Lankan populations irrespective of their gender, age or level of formal education. Literature underscores the importance of using culture free tests with elderly and illiterate populations as they provide less threatening situation in which older people can fully demonstrate their ability independent of language and level of education.

Our study highlights influence of demographic variables on test performance. It also points to limitations and strengths of these tests. Major limitation of this study is limited number of participants.

\section{Acknowledgements}

We wish to thank all the participants of our study and TURIS project of University of Ruhuna (Grant No. APW/SRL/HRH/001/RB/07/ER/1.1.9) for funding the study.

\section{References}

1. Strauss E, Sherman EMS, Spreen O. A compendium of Neuropsychological Tests, $3^{\text {rd }}$ ed. Oxford; University Press: 2006

2. World Bank 2006 Sri Lanka Aging Survey - SLAS, the the survey based on a representative sample of Sri Lankan people. http://siteresources.worldbank.org/ INTSRILANKA/ Resources/LKAging EXxSum.pdf

3. De Silva HA, Gunathilake SB. Mini mental state examination in Sinhalese. A sensitive test to screen for dementia in Sri Lanka. Int J Geriatr Psychiatry, 2002 Feb; 17(2): 134-9.

f. Kathriarachchi ST, Sivayogan S, Jayaratna SD, Dharmasena SR. Comparison of three instruments used in the assessment of dementia in Sri Lanka. Indian JPsychiatry, 2005 Apr; 47(2): 109-12.
5. Umayal S, Kulathunga M, Somaratne S, et al. Validation of a functional screening instrument for dementia in an elderly Sri Lankan population: Comparison of modified Bristol and blessed activities of daily living scales. $B M C$ Res Notes, 2010 Oct; 26(3): 268.

6. Kulathunga M, Umayal S, Somaratne S, Srikanth S, Kathriarachchi S, De Silva K. Validation of the geriatric depression scale for an elderly Sri Lankan clinic population. Indian JPsychiatry $2010 \mathrm{Jul}$; 52(3): 254-6.

7. Karunaratne S, Hanwella R, de Silva V. Validation of the Sinhala version of the Montreal Cognitive Assessment in screening for dementia. Ceylon Medical Journal, 2011 Dec; 56(4): 147-53.

8. Srinivasan S, Jaleel Q. Norms for a neuropsychological test battery to diagnose dementia in the elderly: A study from Sri Lanka. Journal Neurosci Rural Pract, 2015 AprJun; 6(2): 177-81.

9. Suraweera C, Anandakumar D, Dahanayake D, Subendran M, Perera UT, Hanwella R, de Silva VA. Validation of the Sinhala version of the Repeatable Battery for Assessment of Neuropsychological Status (RBANS). Ceylon Medical Journal, 2016 Dec: 61(4): 167-70.

10. Parker $\mathrm{C}$ and Philp I. Screening for cognitive impairment among older people in black and minority ethnic groups. Age and Ageing, 2014; 33 (5).

11. Hogervorst E, Combrinck M, Laputerta P, Rue J, et al. The Hopkins verbal learning test and screening for dementia. Dermet Geriatr Cogn Disord, 2002; 13(1): 13-20.

12. Grober E, Buschke H, Crystal H, Bang S, Dresner R. Screening for dementia by memory testing. Neurology, 1988 Jun; 38(6): 900-3.]

13. Boeve, et. al. Mild cognitive impairment in the oldest old. Neurology, 2003; 60: 477-80.

14. Sivan AB. Benton Visual Retention Test ( $5^{\text {th }}$ ed.) San Antonio Tex: The Psychological Corporation.

15. Brandt J, Benedict RHB. (2001) Hopkins Verbal Learning Test-Revised. Odessa, Fla,: PAR.

16. Kramer $\mathrm{JH}$, et al. Age and gender interactions on verbal memory performance. J Int Neuropsychol Soc, 2003; 9(1): 97-102.

17. Craik FI, Rose NS. Memory encoding and aging: a neurocognitive perspective. Neurosci Biobehav Rev, 2012; 36(7): 1729-39.

18. McDaniel M, Einstein G, Jacoby L. New considerations in aging and memory: the glass may be half full. In: The handbook of aging and cognition. FI Craik, TA Salthouse, Editors. Erlbaum: Mahwah, NJ, 2008; 53(6): 251-310. 